\title{
Spatial transcriptomics: paving the way for tissue-level systems biology
}

\author{
Andreas E. Moor ${ }^{1}$ and Shalev Itzkovitz ${ }^{1}+$ \\ ${ }^{1}$ Department of Molecular Cell Biology, Weizmann Institute of Science, Rehovot, Israel. \\ +To whom correspondence should be addressed. \\ E-mail: shalev.itzkovitz@weizmann.ac.il
}

The field of systems biology integrates mathematical models and large-scale measurements to understand how cellular components cooperatively achieve biological goals. The tissues in our bodies are complex systems composed of diverse cell types that often interact in highly structured repeating anatomical units. External gradients of morphogens, directional blood flow, as well as the secretion and absorption of materials by cells generate distinct microenvironments at different tissue coordinates. Such spatial heterogeneity enables optimized function through division of labor among cells. Unraveling the design principles that govern this spatial division of labor requires techniques to quantify the entire transcriptomes of cells while accounting for their spatial coordinates. In this review we describe how recent advances in spatial transcriptomics open the way for tissuelevel systems biology.

\section{Introduction}

The field of Systems Biology has made a profound impact on our ability to reverse engineer the biological networks that govern cellular behavior. Fueled by biotechnological developments over the past two decades, researchers have been able to obtain a detailed description of regulatory, signaling and metabolic networks 
[1-3]. Remarkably, many of these networks exhibited features that were common to engineered systems, such as modularity, robustness and recurring building blocks [4]. The techniques used for these studies required 'bulk' analyses of extracts from many cells, be it RNA [5, 6], proteins [7] or chromatin [8]. While highly informative for unicellular organisms or cultured cells, these techniques provide only partial information when the biological systems are heterogeneous.

The tissues in our bodies consist of diverse cell types and sub-populations; their molecular identities would be lost in bulk measurements. Single cell RNA sequencing [9-13] has revolutionized our ability to identify and characterize such sub-populations. However, these techniques require tissue dissociation, thus losing the original tissue coordinates. Since the physical location of a cell within the tissue is a key determinant of its molecular identity, tissue-level systems biology requires obtaining whole-genome measurements while accounting for the spatial localization of cells. Several methods for spatial transcriptomics have been comprehensively reviewed in [14], herein we will focus on more recent advances in the field and their potential use in advancing our understanding of design principles of tissue organization.

\section{Spatial context is a key determinant of cellular identity in mammalian tissues}

To motivate the importance of spatial transcriptomics, we will shortly describe open questions in two typical heterogeneous, yet structured mammalian tissues - the intestine and the liver. The mammalian small intestine is lined with a highly folded epithelial sheet composed of deep pits called crypts, and larger protrusions called villi (Figure 1). Stem cells and progenitors within the crypt constantly feed the villus with secretory goblet cells and absorptive enterocytes. These differentiated cells rapidly migrate along the villus walls as they operate for a few days, until they are shed off from the villi tips. The positions of cells along the crypt villus axis correlate 
with their age, making this a classic system to study processes of cell differentiation, homeostasis, aging and death.

Extensive studies deciphered the homeostatic mechanisms that operate in the crypts to maintain constant stem cell numbers while ensuring a steady flux of differentiated cells [15-17]. Much less is known about the diverse processes in the differentiated compartments - the intestinal villi. How fast do enterocytes mature upon entering the villi? Do 'old' enterocytes at the villi tips operate less efficiently than 'young' enterocytes at the villi bottoms as a result of accumulated cellular damage acquired due to the hostile lumen environment (Figure 1A)? Are there different subtypes of enterocytes that sub-specialize in the absorption of particular nutrients, such as carbohydrates, lipids or amino acids, or are enterocytes allabsorbing generalists (Figure 1B)? If such enterocyte division of labor exists is it a result of clonal subtypes generated in the crypts, or rather a transient division of labor modulated by the constantly changing nutrient composition in the gut (Figure 1C)? Are there spatial domains along the vertical villus axis for such sub-populations that define a hierarchy of absorption? Tools to characterize the complete cellular gene expression signatures of enterocytes along the vertical crypt-villus axis would address these open questions and unravel how the intestine can economically yet efficiently absorb nutrients that only exist for transient periods of time [18].

The mammalian liver is a second example in which the spatial locations of cells are critical for understanding their molecular identities and physiological roles. The liver is a central organ for maintaining organismal homeostasis. Hepatocytes perform a wealth of biological tasks ranging from protein secretion, through nutrient storage and release, to detoxification. The hepatocytes that perform these tasks operate in repeating hexagonal anatomical units termed 'lobules'. Each lobule consists of around 12-15 concentric layers of hepatocytes and is polarized by blood that flows inward from outer portal nodes towards draining central veins (Figure 2). The absorption and secretion of hepatocytes residing along the radial blood vessels modulates the microenvironment available for more 'downstream' hepatocytes, creating a fertile ground for tissue optimization. For example, excessive respiration 
or nutrient consumption of portal hepatocytes could potentially deplete these inputs to levels that could dangerously compromise the function of central hepatocytes. Indeed, hepatocytes at different lobule layers sub-specialize in different tasks in a way that is thought to facilitate optimal overall liver function. For example, the outer highly oxygenated portal layers of the lobules sub-specialize in energy demanding tasks such as protein secretion and glucose production, whereas central hepatocytes specialize in detoxification $[19,20]$. Obtaining the complete gene expression signatures of individual hepatocytes at all lobule coordinates would unravel the design principles underlying this spatial division of labor and facilitate detailed modeling of the liver response to diverse perturbations.

\section{In situ spatial transcriptomics}

The age of spatial transcriptomics can be traced back to the development of single molecule fluorescence in situ hybridization (smFISH) methods [21, 22]. smFISH enables transcript quantification in situ (at the tissue site where they reside) by making use of libraries of multiple short, $20 \mathrm{bp}$ long olignonucleotide probes, each labeled with typically a single fluorophore (Figure 3A1). Through the specific accumulation of these fluorescent probes on the target mRNA, individual transcripts can be visualized as diffraction-limited spots by fluorescence microscopy [22]. smFISH has been applied in cells as well as in mammalian tissues [23], where it enabled characterization of cell sub-populations and spatial heterogeneity [24-26].

An outstanding limitation of smFISH is the small number of transcripts that can simultaneously be identified, typically three or four. This is due to the limited number of fluorophores that are suitable for parallel use. An elegant approach to overcome this limitation uses combinatorial labeling. Levsky et al. [27] were the first to demonstrate combinatorial labeling for single transcript detection. By dividing the library of probes into groups, each coupled to one of the three fluorophores, they could differentiate between dots that have single colors, pairs of colors or triplets. This approach increased the number of transcripts that could be 
detected with $\mathrm{n}$ fluorophores to $2^{\mathrm{n}}-1$. Lubeck and Cai further increased the number of simultaneously detected transcripts by combining spatial barcodes with superresolution microscopy [28]. With the higher resolution of $10-20 \mathrm{~nm}$ they were able to resolve different physical sub-parts of the mRNA molecules of interest (Figure $3 \mathrm{~A} 2$ ). In this way the transcripts of two different genes combinatorially labeled with three colors could be resolved if one uses a sequence of probes coupled to red, blue and green, whereas the other uses a sequence of blue, red and green. An additional factor that increased the number of simultaneously detected genes was the use of seven photoswitchable dye pairs, as compared to the four fluorescent dyes typically detected in smFISH experiments.

An alternative method for combinatorial smFISH uses the temporal dimension to multiplex probe libraries. Cai and colleagues generated four different versions of a smFISH probe library, each with an identical probe set [29]. Each library was coupled to one of four fluorophores. During each hybridization round only one of the four libraries was utilized, and DNAse treatements removed bound probes between hybridization rounds (Figure 3A3). The recorded sequence of probe signals enabled the identification of the detected RNA species based on the temporal sequence of dots. In theory, this approach allows for the detection of $\mathrm{F}^{\wedge} \mathrm{n}$ distinct transcripts ( $\mathrm{F}=$ fluorophores, $\mathrm{n}=$ hybridiaztion rounds)[29]. For example, with 4 fluorophores and 8 hybridization rounds one could identify 65,536 different transcripts. However, this approach would currently be prohibitively expensive if applied transcriptome-wide.

A pending issue with combinatorial smFISH is the robustness to readout errors. Since probe hybridization is an inherently stochastic process, one could erroneously assign an RNA species to the wrong gene if one of the sequential hybridizations failed. This readout error increases exponentially with the number of hybridization rounds. To overcome this, Chen et al. developed an elegant barcode assignment scheme termed 'MERFISH', which ensures that the sequence of barcodes for different genes is far enough so that only multiple readout errors would cause miss-assignments [30, 31]. In addition, both the cost of fluorescent probe synthesis 
and time-consuming hybridization rounds were addressed by MERFISH through a clever two-stage hybridization scheme (Figure 3A4), leading to the accurate detection of 1000 transcripts with 14 hybridization rounds in 100 cells [30]. A recent improvement of MERFISH enabled the detection of 130 genes in 40,000 cells in one 18h experiment [32]. These proof-of concept studies for combinatorial smFISH were applied to cultured single cells. It remains to be seen how well the methods will perform in tissue sections. This could be particularly challenging since tissues have an inherently larger readout noise due to increased auto-fluorescence.

\section{In-situ sequencing}

While combinatorial methods have dramatically increased the throughput of in-situ transcript detection, they are still targeted approaches, requiring pre-selecting genes of interest for which probes are designed. In-situ sequencing is a complementary technique that enables an unbiased census of all RNA molecules while preserving localization. High-throughput sequencing reconstructs the sequence of cDNA molecules tethered to a flow cell by sequential synthesis with fluorescent nucleotides. In situ RNA sequencing in essence replaces the flow cell with the original tissue of interest (Figure 3B). It utilizes in situ cDNA synthesis, cDNA amplification and cross-linking [33-35]. The resulting circular cDNA amplicons are amenable to SOLID sequencing by ligation; four-color microscopy records the SOLID base information for each pixel, the fluorophore is inactivated and the sequencing reaction is repeated for the next base. Nilsson and colleagues introduced targeted in situ RNA sequencing by making use of padlock probes to initiate targeted cDNA synthesis in situ [33]. They sequenced four base-pair fragments and detected 31 different transcripts in parallel in fixed tissue sections. Lee et al. further developed Fluorescent in situ RNA sequencing (FISSEQ) by generating 150,000 short $30 \mathrm{bp}$ reads that were mapped to 8,100 genes in fibroblasts. They also demonstrated the in situ cDNA library generation in intact tissues, such as drosophila embryos and mouse brain sections. Some inconsistencies 
in the correlations between FISSEQ reads and traditional RNAseq remain to be further explored [35].

A recent promising in-situ sequencing method was developed by Stahl et al. They used a glass slide that spatially captures mRNA for library assembly when overlaid with a tissue section [36]. Each polyA-capturing feature consists of a positional barcode, unique molecular identifier (UMI), and library adaptor sequences (Figure 3C). RNA is eluted and cDNA synthesis and library preparation are performed in bulk reactions for the whole slide. Importantly, spatial information is preserved by the positional barcodes. This results in a considerably simpler workflow compared to FISSEQ and sequential FISH. The method currently utilizes 1000 capture features of $100 \mu \mathrm{m}$ diameter, spread across a $6 \times 6 \mathrm{~mm}$ area. It is a promising approach that could enable non-specialized laboratories to robustly perform spatial transcriptomics in tissues, albeit with a currently low resolution and spatial coverage.

Both sequential FISH and FISSEQ methods suffer from densities of labeled transcripts that surpass the resolution capacity of the optical system. Due to the diffraction limit of $\sim 0.3 \mathrm{um}$, a typical mammalian cell with a diameter of $10 \mathrm{um}$ would only contain on the order of $10^{4}$ 'pixels'. Since there are around $10^{6} \mathrm{mRNA}$ molecules per cell, each pixel thus contains about 100 mRNA molecules, rendering efficient optical detection impossible. To overcome this limitation Coskun and Cai have applied local image correlation patterns to quantify highly abundant transcripts with conventional fluorescence microscopy in both cultured single cells and tissue sections [37]. An alternative approach to saturated transcript density consists of tissue expansion. Expansion FISH (ExFISH) links RNA molecules to a swellable gel and enables effectively super resolution RNA imaging with diffractionlimited microscopes in thick tissues [38]. The combination of ExFISH with selfamplifying smFISH probes [39] led to bright dots in thick tissues that can be rapidly acquired with lightsheet microscopy [38]. 


\section{Combination of single cell RNA sequencing with tissue reference maps}

A complementary approach for obtaining spatial transcriptomics is to infer the tissue coordinate from the single cell expression patterns of dissociated tissues (Figure 3D). Single cell RNA sequencing (scRNAseq) methods generate simultaneous gene expression data of thousands of cells [9-13]. A given cell's positional origin within the tissue of interest is lost, however, during the dissociation process. Several laboratories have utilized pre-established tissue reference maps, which consist of the spatial expression patterns of a selected subset of marker genes, to infer the positional information of cells in the scRNAseq data (Figure 3D). The laboratory of Stefan Heller generated single cell qRT-PCR data of several hundred sorted otocyst cells [40]. Subsequently they used expression patterns of a few genes that were previously mapped out using RNA in-situ hybridization, to establish a threedimensional model of the otocyst in the shape of a sphere. This elegant approach enabled mapping the single cell expression dataset back to its positional origin by using 3D principal component analysis [40]. Ensuing studies generated in situ maps and used them to infer the position of scRNAseq data in the zebrafish embryo [41], the developing brain of a bristle worm [42], and gastrulating mouse embryos [43, 44].

A recent study combined scRNAseq with tissue reference maps that were generated with smFISH to reconstruct the global spatial expression profiles along the mammalian liver lobule [45]. The higher precision and dynamic range of smFISH, compared to traditional in-situ hybridization techniques enables precise spatial inference with significantly smaller number of marker genes. The use of smFISH to create tissue reference maps becomes critical in tissues where genes are expressed in a graded, rather than in a binary manner. The tissue reference mapbased methods combine the advantages of two worlds: the ability of sequencing methods to perform deep unbiased profiling of large amounts of single cells and preservation of positional information of in situ methods. Central to these methods 
are sophisticated algorithms for inferring the positional information while accounting for different experimental sources of variability.

\section{Theory and outlook}

The diverse methods for spatial transcriptomics are expected to generate highly detailed maps of single cell gene expression at any tissue coordinate. As with other previous revolutions in systems biology we expect a parallel development of theoretical frameworks to integrate these measurements and identify tissue design principles. One central goal of mammalian systems biology is to understand a tissue's metabolic response to diverse inputs. Large-scale reconstructions of liver metabolic networks [46] enable simulating such responses, however these networks model the liver as a homogenous well-mixed pool of cells. Given the intricate spatial division of labor along the liver lobule radial axis there is a need to develop metabolic modeling approaches that will consist of connected cellular subnetworks, which exchange substrates and products [47-49]. Key to the success of these methods is an integration of the single cell spatial measurements with advanced tissue imaging techniques to define the interactions between the relevant tissue cellular components [50,51].

A second avenue to explore is the modeling of how cell circuits achieve tissue homeostasis. Interactions between cells in a tissue, through juxtacrine and paracrine signaling, are crucial for tuning overall tissue function and for maintaining homeostatic balance of biomass. Theoretical works that analyze the performance of diverse cell circuits will be instrumental to understanding how cells in tissues jointly achieve physiological goals [52-55]. Another exciting theoretical avenue is the analysis of the conditions when division of labor among cells is more optimal for organ function compared to homogenous tissues with generalist cells. This question has analogies in ecology, economics and engineering, e.g. the mathematical analysis of castes systems in social insect colonies [56], and the theory of pareto optimality $[57,58]$. The astonishing speed of current developments in imaging and sequencing 
technologies promises better resolution, better transcriptome coverage and cheaper assays in the near future. Integrating this flood of high quality data with theory will dramatically advance the field of tissue systems biology and could expose the underlying principles of tissue organization.

\section{Figure legends}

\section{Figure 1}

Open questions in the biology of the intestinal epithelium that require spatially resolved single-cell measurements. This illustration depicts a small intestinal cryptvillus unit. Each crypt forms a well-protected adult stem cell niche. Stem cells at the bottom of the crypt, intermingled with supporting Paneth cells, constantly proliferate to generate progenitors, termed 'transit amplifying cells'. These migrate upwards as they continue to divide yielding a constant flux of differentiated secretory goblet cells and nutrient absorbing enterocytes that feed the villi. The differentiated cells migrate upwards along the villi walls as they function for a few days until they are shed off from the villi tops. Several open questions regarding the collective behavior of this key cell population include: A - Are 'old' enterocytes, that have arrived at the tip of the villus, functionally different from 'young' enterocytes that only recently acquired their differentiated function? B - Are enterocytes "generalists" that absorb each nutrient class equally efficiently or are there subspecialized types of enterocytes that preferably absorb carbohydrates, lipids or amino acids? C - If such sub-specialized enterocytes exist, do they differentiate hierarchically from stem cells and maintain their fate in a stable manner? Alternatively, the fractions of these sub-specialized cells might fluctuate dynamically depending on extrinsic stimuli, e.g. the presence or absence of the respective nutrients. Quantitative measurements of the complete transcriptome of enterocytes at defined coordinates along the villus axis can address these and other open questions. 


\section{Figure 2}

Unresolved questions regarding the spatial organization of the liver. The liver is orchestrating anabolism and catabolism of nutrients and xenobiotics. It is composed of hexagonal lobules that are polarized by blood flow; Blood originates from portal triads at the corners of the lobule and flows towards a draining central vein. Each portal triad consists of a hepatic artery (red) that carries oxygenated blood, a portal vein (blue) that conveys nutrient-rich blood from the gastrointestinal tract and a bile duct (green). Gray arrows indicate directions of flow, yellow arrows indicate exchange of materials between hepatocytes and blood, green arrows mark bile canaliculi. Various metabolic tasks are spatially distributed in specialized sublobular layers between the central vein and the portal field, an organization termed "liver zonation". Where along the radial lobule axis is each liver function localized? What are the optimality principles governing this spatial division of labor? Are there spatially distributed metabolic cycles in which pathway intermediates are transferred among sequential cells? Spatially resolved transcriptomics of the liver could facilitate detailed understanding of this fundamental organ.

\section{Figure 3}

Methods for spatial transcriptomics. A: In situ hybridization methods. 1) single molecule RNA fluorescence in situ hybridization enables identifying individual transcripts as fluorescent dots, by making use of libraries of multiple $20 \mathrm{bp}$ long olignonucleotide probes, each labeled with a single fluorophore .2) The use of super resolution microscopy in combination with smFISH stainings enables the identification of fluorophore sequences along single transcripts [28]. Multiple transcripts can be identified by making use of differing fluorophore orders. 3) Temporal barcoding is based on sequential hybridizations and imaging steps that are followed by the digestion of the imaged probe olignonucleotides. Subsequent 
reactions make use of probe sets that are labeled with differing fluorophores, the resulting temporal barcode of fluorophores for each smFISH dot enable the identification of multiple transcripts [29]. 4) The MERFISH approach makes use of a two-stage hybridization scheme [30-32]. Encoding probes are hybridized to RNA molecules in situ, these probes encompass landing sequences for the rapid subsequent assembly of fluorescent readout probes. The fluorescent dyes that are coupled to the readout probes can be deactivated chemically and allow for the repeated hybridization cycles with different readout probes. The rapid hybridization cycle duration of this protocol allows for a sophisticated barcode assignment scheme that requires 4 readout errors for barcode miss-assignments; single errors can be unequivocally corrected. B: Fluorescent in situ sequencing (FISSEQ) [34, 35] 1) mRNA molecules are reverse-transcribed in situ and circularized. 2) Resulting cDNA molecules are amplified with rolling circle amplification. Cross-linking of modified cDNA residues prevents diffusion of the resulting amplicons. 3) Repeated sequencing reactions by ligation and subsequent microscopic imaging and fluorophore cleavage yield a nucleotide sequence for each imaged pixel; sequences that are retrieved from RNA molecules can be mapped to a reference transcriptome while background fluorescence is not retrieved. C: Spatial mRNA capture slide [36] 1) Commercially available mRNA capturing slides incorporate polyT-nucleotides with embedded positional barcodes. 2) Tissue sections are permeabilized and mRNA molecules are captured by the polyTnucleotides of the glass slide. 3) Captured mRNA molecules are eluted from the glass slide and feature a positional barcode. Subsequent library preparation and massively parallel sequencing enables the retrieval of the corresponding positional barcode and originating tissue coordinates for each resulting library. D: Reference map-based single cell RNA sequencing. 1) Spatial expression patterns of landmark genes are mapped in 2 or 3 dimensional space within the intact tissue. 2) In parallel, the tissue of interest is dissociated into single cells; these cells are profiled in depth by scRNAseq [41-45] or single cell qPCR [40]. 3) The expression levels of the landmark genes that were used for the assembly of positional tissue maps in 1) are retrieved for all sequenced single cells. 4) The original position of the sequenced 
single cells is inferred based on their expression levels of all marker genes. This consequently enables identifying the spatial domain of expression of any gene of interest.

\section{Acknowledgements}

We thank Shani Ben-Moshe for valuable comments on the manuscript. A.E.M. is supported by the Swiss National Science Foundation (grant 158999) and the EMBO Long -Term Fellowship program (ALTF 306-2016). S.I. is supported by the Henry Chanoch Krenter Institute for Biomedical Imaging and Genomics, The Leir Charitable Foundations, Richard Jakubskind Laboratory of Systems Biology, Cymerman-Jakubskind Prize, The Lord Sieff of Brimpton Memorial Fund, the I-CORE program of the Planning and Budgeting Committee and the Israel Science Foundation (grants 1902/ 12 and 1796/12), the Israel Science Foundation grant No. 1486/16, the EMBO Young Investigator Program and the European Research Council under the European Union's Seventh Framework Programme (FP7/20072013)/ERC grant agreement number 335122. S.I. is the incumbent of the Philip Harris and Gerald Ronson Career Development Chair.

\section{References}

- $\quad$ of special interest

- $\quad$ of outstanding interest

1. Karlebach, G., \& Shamir, R. (2008). Modelling and analysis of gene regulatory networks. Nature Reviews Molecular Cell Biology, 9(10), 770-780. doi:10.1038/nrm2503 
2. Papin, J. A., Hunter, T., Palsson, B. O., \& Subramaniam, S. (2005). Reconstruction of cellular signalling networks and analysis of their properties. Nature Reviews Molecular Cell Biology, 6(2), 99-111. doi:10.1038/nrm1570

3. Guimerà, R., \& Nunes Amaral, L. A. (2005). Functional cartography of complex metabolic networks. Nature, 433(7028), 895-900. doi:10.1038/nature03288

4. Alon, U. (2003). Biological Networks: The Tinkerer as an Engineer. Science, 301(5641), 1866-1867. doi:10.1126/science.1089072

5. Duggan, D. J., Bittner, M., Chen, Y., Meltzer, P., \& Trent, J. M. (1999). Expression profiling using cDNA microarrays. Nature Genetics, 21, 10-14. doi:10.1038/4434

6. Wang, Z., Gerstein, M., \& Snyder, M. (2009). RNA-Seq: A revolutionary tool for transcriptomics. Nature Reviews Genetics, 10(1), 57-63. doi:10.1038/nrg2484

7. Aebersold, R., \& Mann, M. (2003). Mass spectrometry-based proteomics. Nature, 422(6928), 198-207. doi:10.1038/nature01511

8. Furey, T. S. (2012). ChIPseq and beyond: New and improved methodologies to detect and characterize protein interactions. Nature Reviews Genetics, 13(12), 840852. doi:10.1038/nrg3306

9. Hashimshony, T., Wagner, F., Sher, N., \& Yanai, I. (2012). CEL-Seq: Single-Cell RNASeq by Multiplexed Linear Amplification. Cell Reports, 2(3), 666-673. doi:10.1016/j.celrep.2012.08.003

10. Jaitin, D. A., Kenigsberg, E., Keren-Shaul, H., Elefant, N., Paul, F., Zaretsky, I., ... Amit, I. (2014). Massively parallel single-cell RNA-seq for marker-free decomposition of tissues into cell types. Science, 343(6172), 776-9. doi:10.1126/science.1247651

11. Zeisel, A., Muñoz-Manchado, A. B., Codeluppi, S., Lönnerberg, P., La Manno, G., Juréus, A., ... Linnarsson, S. (2015). Brain structure. Cell types in the mouse cortex 
and hippocampus revealed by single-cell RNA-seq. Science, 347(6226), 1138-1142. doi:10.1126/science.aaa1934

12. Klein, A. M., Mazutis, L., Akartuna, I., Tallapragada, N., Veres, A., Li, V., ... Kirschner, M. W. (2015). Droplet Barcoding for Single-Cell Transcriptomics Applied to Embryonic Stem Cells. Cell, 161(5), 1187-1201. doi:10.1016/j.cell.2015.04.044

13. Macosko, E. Z., Basu, A., Satija, R., Nemesh, J., Shekhar, K., Goldman, M., ... McCarroll, S. A. (2015). Highly Parallel Genome-wide Expression Profiling of Individual Cells Using Nanoliter Droplets. Cell, 161(5), 1202-1214. doi:10.1016/j.cell.2015.05.002

14. Crosetto, N., Bienko, M., \& van Oudenaarden, A. (2015). Spatially resolved transcriptomics and beyond. Nature Reviews Genetics, 16(1), 57-66. doi:10.1038/nrg3832

15. Barker, N. (2014). Adult intestinal stem cells: Critical drivers of epithelial homeostasis and regeneration. Nat Rev Mol Cell Biol, 15(1), 19-33. doi:10.1038/nrm3721

16. Crosnier, C., Stamataki, D., \& Lewis, J. (2006). Organizing cell renewal in the intestine: Stem cells, signals and combinatorial control. Nat Rev Genet, 7(5), 349-59. doi: $10.1038 / \operatorname{nrg} 1840$

17. Tóth, B., Ben-Moshe, S., Gavish, A., Barkai, N., \& Itzkovitz, S. (2017). Early commitment and robust differentiation in colonic crypts. Mol Syst Biol,13. doi: $10.15252 / \mathrm{msb} .20167283$

18. Ferraris, R. P., \& Diamond, J. M. (1989). Specific Regulation of Intestinal Nutrient Transporters by their Dietary Substrates. Annual Review of Physiology, 51(1), 125141. doi:10.1146/annurev.ph.51.030189.001013

19. Jungermann, K., \& Kietzmann, T. (1996). Zonation of parenchymal and nonparenchymal metabolism in liver. Annual Review of Nutrition, 16, 179-203. doi:10.1146/annurev.nu.16.070196.001143 
20. Gebhardt, R., \& Matz-Soja, M. (2014). Liver zonation: Novel aspects of its regulation and its impact on homeostasis. World Journal of Gastroenterology : WJG, 20(26), 8491-8504. doi:10.3748/wjg.v20.i26.8491

21. Levsky, J. M., \& Singer, R. H. (2003). Fluorescence in situ hybridization: Past, present and future. Journal of Cell Science, 116(14), 2833-2838. doi:10.1242/jcs.00633

22. Raj, A., van den Bogaard, P., Rifkin, S. A., van Oudenaarden, A., \& Tyagi, S. (2008). Imaging individual mRNA molecules using multiple singly labeled probes. Nat Methods, 5(10), 877-9. doi:10.1038/nmeth.1253

23. Itzkovitz, S., \& van Oudenaarden, A. (2011). Validating Transcripts with Probes and Imaging Technology. Nature methods, $8(4$ Suppl), S12-S19. doi:10.1038/nmeth.1573

24. Itzkovitz, S., Lyubimova, A., Blat, I. C., Maynard, M., van Es, J., Lees, J., ... van Oudenaarden, A. (2012). Single-molecule transcript counting of stem-cell markers in the mouse intestine. Nat Cell Biol, 14(1), 106-14. doi:10.1038/ncb2384

25. Lyubimova, A., Itzkovitz, S., Junker, J. P., Fan, Z. P., Wu, X., \& van Oudenaarden, A. (2013). Single-molecule mRNA detection and counting in mammalian tissue. Nature Protocols, 8(9), 1743-1758. doi:10.1038/nprot.2013.109

26. Bahar Halpern, K., Tanami, S., Landen, S., Chapal, M., Szlak, L., Hutzler, A., ... Itzkovitz, S. (2015). Bursty gene expression in the intact mammalian liver. Molecular Cell, 58(1), 147-156. doi:10.1016/j.molcel.2015.01.027

27. Levsky, J. M., Shenoy, S. M., Pezo, R. C., \& Singer, R. H. (2002). Single-cell gene expression profiling. Science, 297(5582), 836-840. doi:10.1126/science.1072241

- This work introduced spectral barcoding of single molecule FISH probes to increase the number of targets that can be simultaneously identified. 
28. Lubeck, E., \& Cai, L. (2012). Single-cell systems biology by super-resolution imaging and combinatorial labeling. Nature Methods, 9(7), 743-748. doi:10.1038/nmeth.2069

- $\quad$ This work scales up the number of simultaneously detected transcripts by using super-resolution microscopy, resolving the spatial order of differentially labeled probe sets along the transcripts of interest.

29. Lubeck, E., Coskun, A. F., Zhiyentayev, T., Ahmad, M., \& Cai, L. (2014). Single-cell in situ RNA profiling by sequential hybridization. Nature Methods, 11(4), 360-361. doi:10.1038/nmeth.2892

30. Chen, K. H., Boettiger, A. N., Moffitt, J. R., Wang, S., \& Zhuang, X. (2015). RNA imaging. Spatially resolved, highly multiplexed RNA profiling in single cells. Science, 348(6233), aaa6090. doi:10.1126/science.aaa6090

-. The authors present MERFISH, a method that enables the rapid detection of hundreds of transcripts with a two-stage hybridization protocol. The method incorporates an error-correction mechanism for barcode miss-assignments.

31. Moffitt, J. R., \& Zhuang, X. (2016). Chapter One - RNA Imaging with Multiplexed Error-Robust Fluorescence In Situ Hybridization (MERFISH). In G. S. F. Jaffrey \& S. R. (Eds.), Methods in Enzymology (Vol. 572, pp. 1-49). Academic Press.

32. Moffitt, J. R., Hao, J., Wang, G., Chen, K. H., Babcock, H. P., \& Zhuang, X. (2016). High-throughput single-cell gene-expression profiling with multiplexed errorrobust fluorescence in situ hybridization. Proceedings of the National Academy of Sciences, 201612826. doi:10.1073/pnas.1612826113

33. Ke, R., Mignardi, M., Pacureanu, A., Svedlund, J., Botling, J., Wählby, C., and Nilsson, M. (2013). In situ sequencing for RNA analysis in preserved tissue and cells. Nature Methods, 10, 857-860. doi: 10.1038/nmeth.2563 
34. Lee, J. H., Daugharthy, E. R., Scheiman, J., Kalhor, R., Yang, J. L., Ferrante, T. C., ... Church, G. M. (2014). Highly multiplexed subcellular RNA sequencing in situ. Science, 343(6177), 1360-1363. doi:10.1126/science.1250212

- $\quad$ This study describes in situ cDNA synthesis followed by sequencing by ligation in cells and tissues. Corresponding mRNA sequences are microscopically identified for each pixel and directly yields in subcellular spatial transcriptomic profiles.

35. Lee, J. H., Daugharthy, E. R., Scheiman, J., Kalhor, R., Ferrante, T. C., Terry, R., ... Church, G. M. (2015). Fluorescent in situ sequencing (FISSEQ) of RNA for gene expression profiling in intact cells and tissues. Nature Protocols, 10(3), 442-458. doi:10.1038/nprot.2014.191

36. Ståhl, P. L., Salmén, F., Vickovic, S., Lundmark, A., Navarro, J. F., Magnusson, J., ... Frisén, J. (2016). Visualization and analysis of gene expression in tissue sections by spatial transcriptomics. Science, 353(6294), 78-82. doi:10.1126/science.aaf2403

- Ståhl et al. introduce a commercially available glass slide for in situ RNA capturing and positional library barcoding in overlaid tissue sections. This technique brings spatial transcriptomics within reach for laboratories that are not proficient in high-throughput microscopy.

37. Coskun, A. F., \& Cai, L. (2016). Dense transcript profiling in single cells by image correlation decoding. Nature Methods, advance online publication. doi:10.1038/nmeth.3895

38. Chen, F., Wassie, A. T., Cote, A. J., Sinha, A., Alon, S., Asano, S., ... Boyden, E. S. (2016). Nanoscale imaging of RNA with expansion microscopy. Nature Methods, 13(8), 679-684. doi:10.1038/nmeth.3899

- $\quad$ This recent publication features expansion microscopy for RNA imaging. It makes use of expandable gels to achieve super resolution analyses with diffraction-limited microscopes in thick tissues. 
39. Choi, H. M. T., Beck, V. A., \& Pierce, N. A. (2014). Next-generation in situ hybridization chain reaction: Higher gain, lower cost, greater durability. ACS nano, 8(5), 4284-4294. doi:10.1021/nn405717p

40. Durruthy-Durruthy, R., Gottlieb, A., Hartman, B. H., Waldhaus, J., Laske, R. D., Altman, R., \& Heller, S. (2014). Reconstruction of the mouse otocyst and early neuroblast lineage at single-cell resolution. Cell, 157(4), 964-978. doi:10.1016/j.cell.2014.03.036

41. Satija, R., Farrell, J. A., Gennert, D., Schier, A. F., \& Regev, A. (2015). Spatial reconstruction of single-cell gene expression data. Nature Biotechnology, 33(5), 495-502. doi:10.1038/nbt.3192

42. Achim, K., Pettit, J.-B., Saraiva, L. R., Gavriouchkina, D., Larsson, T., Arendt, D., \& Marioni, J. C. (2015). High-throughput spatial mapping of single-cell RNA-seq data to tissue of origin. Nature Biotechnology, 33(5), 503-509. doi:10.1038/nbt.3209

-. $\quad$ References [39-41] pioneered the use of landmark genes to positionally map single-cell expression data back to its spatial tissue coordinates of origin.

43. Peng, G., Suo, S., Chen, J., Chen, W., Liu, C., Yu, F., ... Jing, N. (2016). Spatial Transcriptome for the Molecular Annotation of Lineage Fates and Cell Identity in Mid-gastrula Mouse Embryo. Developmental Cell, 36(6), 681-697. doi:10.1016/j.devcel.2016.02.020

44. Scialdone, A., Tanaka, Y., Jawaid, W., Moignard, V., Wilson, N. K., Macaulay, I. C., ... Göttgens, B. (2016). Resolving early mesoderm diversification through single-cell expression profiling. Nature, 535(7611), 289-293. doi:10.1038/nature18633

45. Halpern, K.B., Shenhav, R., Matcovitch-Natan, O., Tóth, B., Lemze, D., Golan, M., Massasa, E.E., Baydatch, S., Landen, S., Moor, A.E., Brandis, A., Giladi, A., StokarAvihail, A., David, E., Amit, I., \& Itzkovitz, S. (2017). Single-cell spatial reconstruction reveals global division of labour in the mammalian liver. Nature, advance online publication. doi:10.1038/nature21065 
- $\quad$ This paper combined scRNAseq with tissue reference maps established with smFISH to reconstruct the global spatial division of labor in the mammalian liver.

46. Gille, C., Bölling, C., Hoppe, A., Bulik, S., Hoffmann, S., Hübner, K., ... Holzhütter, H.G. (2010). HepatoNet1: A comprehensive metabolic reconstruction of the human hepatocyte for the analysis of liver physiology. Molecular Systems Biology, 6, 411. doi:10.1038/msb.2010.62

47. Holzhütter, H.-G., Drasdo, D., Preusser, T., Lippert, J., \& Henney, A. M. (2012 MayJun2012 May-Jun). The virtual liver: A multidisciplinary, multilevel challenge for systems biology. Wiley Interdisciplinary Reviews. Systems Biology and Medicine, 4(3), 221-235. doi:10.1002/wsbm.1158

48. Harcombe, W. R., Riehl, W. J., Dukovski, I., Granger, B. R., Betts, A., Lang, A. H., ... Segrè, D. (2014). Metabolic resource allocation in individual microbes determines ecosystem interactions and spatial dynamics. Cell Reports, 7(4), 1104-1115. doi:10.1016/j.celrep.2014.03.070

49. Bartl, M., Pfaff, M., Ghallab, A., Driesch, D., Henkel, S. G., Hengstler, J. G., ... Li, P. (2015). Optimality in the zonation of ammonia detoxification in rodent liver. Archives of Toxicology, 89(11), 2069-2078. doi:10.1007/s00204-015-1596-4

50. Morales-Navarrete, H., Segovia-Miranda, F., Klukowski, P., Meyer, K., Nonaka, H., Marsico, G., ... Kalaidzidis, Y. (2015). A versatile pipeline for the multi-scale digital reconstruction and quantitative analysis of 3D tissue architecture. elife, 4. doi:10.7554/eLife.11214

51. Hoehme, S., Brulport, M., Bauer, A., Bedawy, E., Schormann, W., Hermes, M., ... Drasdo, D. (2010). Prediction and validation of cell alignment along microvessels as order principle to restore tissue architecture in liver regeneration. Proceedings of 
the National Academy of Sciences of the United States of America, 107(23), 1037110376. doi:10.1073/pnas.0909374107

52. Youk, H., \& Lim, W. A. (2014). Secreting and sensing the same molecule allows cells to achieve versatile social behaviors. Science, 343(6171), 1242782. doi:10.1126/science.1242782

53. Hart, Y., Reich-Zeliger, S., Antebi, Y. E., Zaretsky, I., Mayo, A. E., Alon, U., \& Friedman, N. (2014). Paradoxical Signaling by a Secreted Molecule Leads to Homeostasis of Cell Levels. Cell, 158(5), 1022-1032. doi:10.1016/j.cell.2014.07.033

54. Lander, A. D., Gokoffski, K. K., Wan, F. Y. M., Nie, Q., \& Calof, A. L. (2009). Cell Lineages and the Logic of Proliferative Control. PLOS Biol, 7(1), e1000015. doi:10.1371/journal.pbio.1000015

55. Jiménez, A., Cotterell, J., Munteanu, A., \& Sharpe, J. (2015). Dynamics of gene circuits shapes evolvability. Proceedings of the National Academy of Sciences of the United States of America, 112, 2103-2108. doi:10.1073/pnas.1411065112

56. Oster, G. F., \& Wilson, E. O. (1978). Caste and ecology in the social insects. Monographs in Population Biology, 12, 1-352.

57. Nagrath, D., Avila-Elchiver, M., Berthiaume, F., Tilles, A. W., Messac, A., \& Yarmush, M. L. (2007). Integrated Energy and Flux Balance Based Multiobjective Framework for Large-Scale Metabolic Networks. Annals of Biomedical Engineering, 35(6), 863-885. doi:10.1007/s10439-007-9283-0

58. Hart, Y., Sheftel, H., Hausser, J., Szekely, P., Ben-Moshe, N. B., Korem, Y., ... Alon, U. (2015). Inferring biological tasks using Pareto analysis of high-dimensional data. Nature Methods, 12(3), 233-235. doi:10.1038/nmeth.3254 
(O) Specialized amino-acid enterocyte

Generalist absorption

Specialized carbohydrate enterocyte

A

Old versus young enterocytes

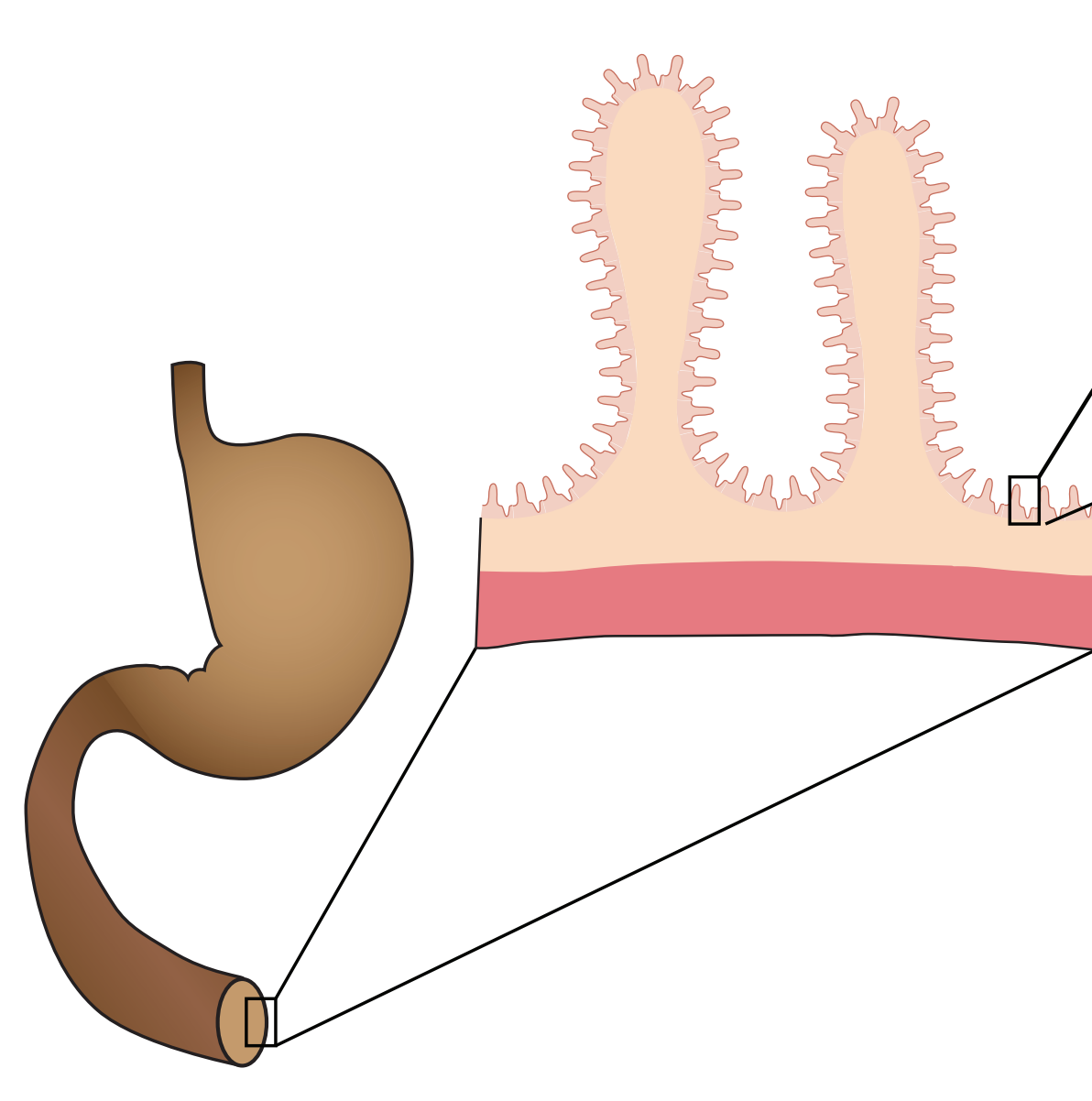

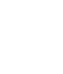

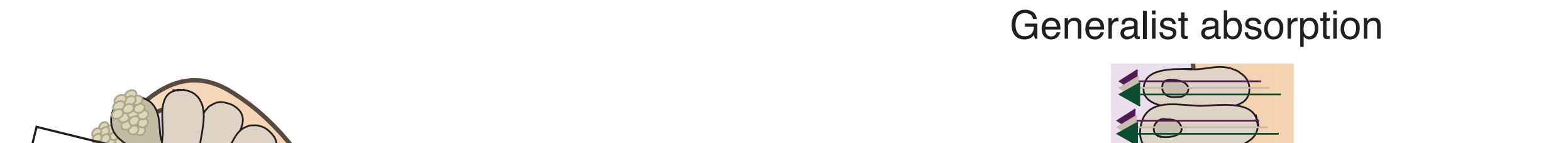

A
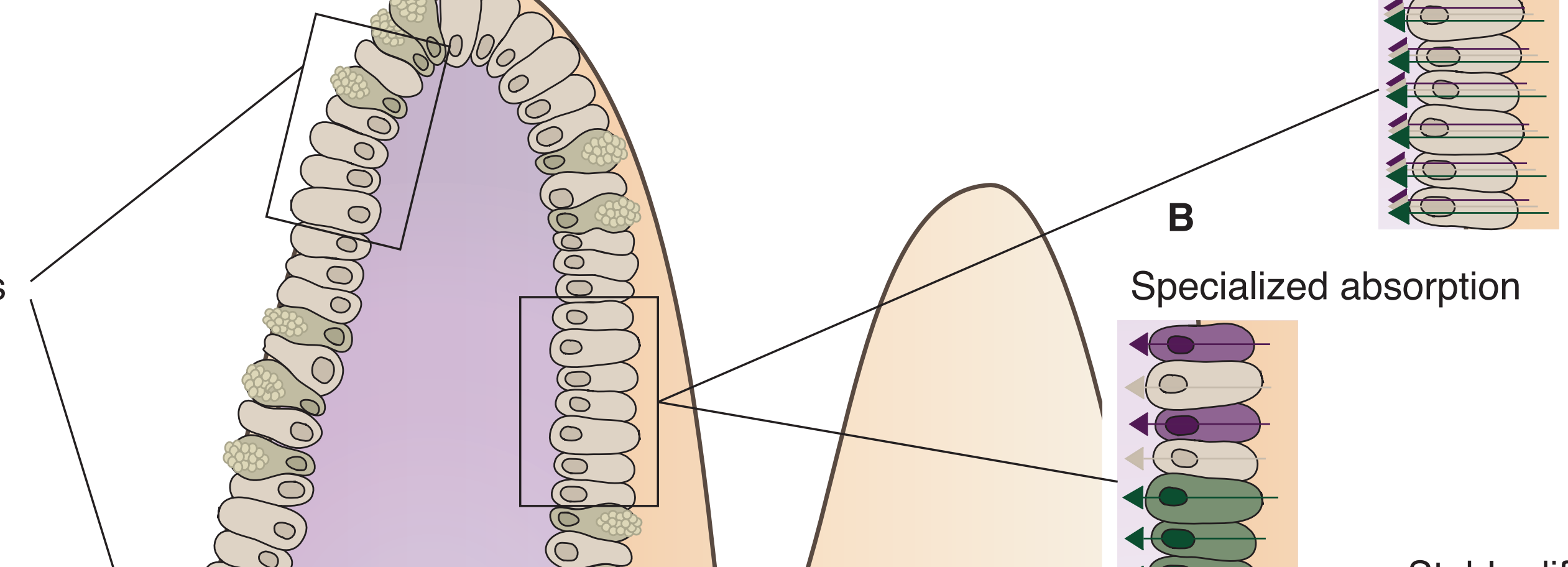

Specialized absorption

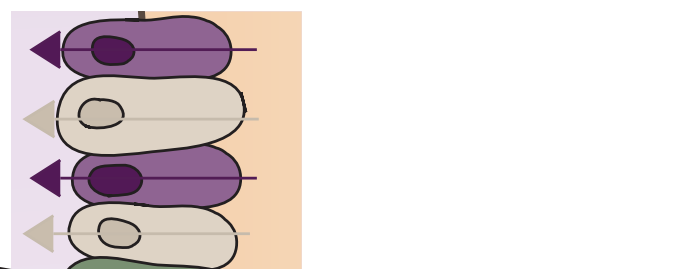




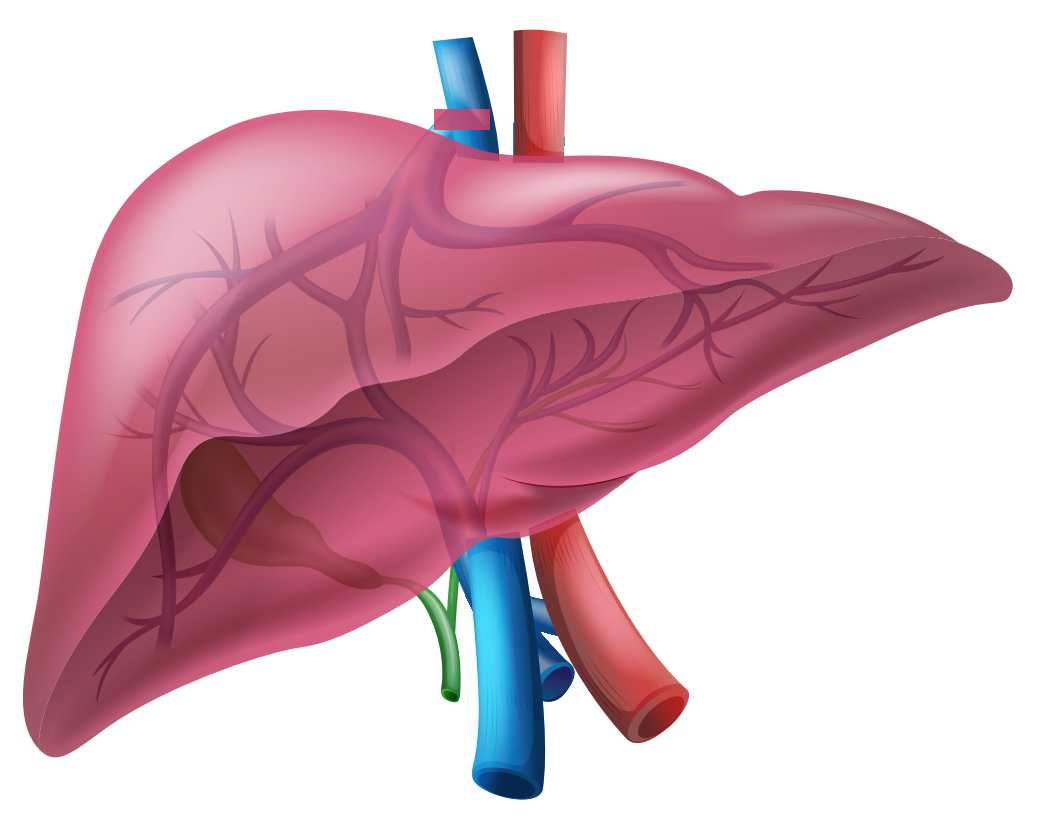

Liver lobule

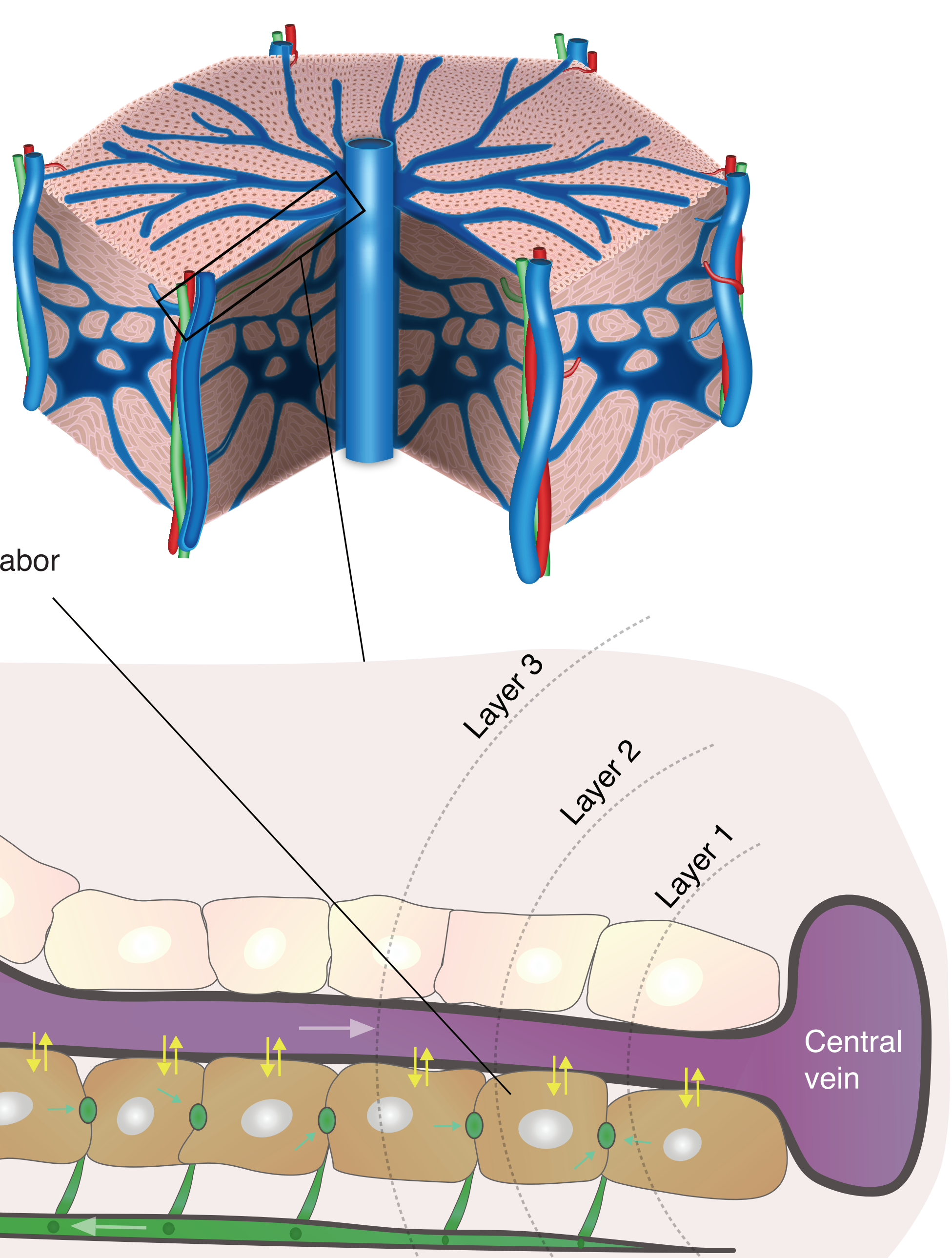


1)

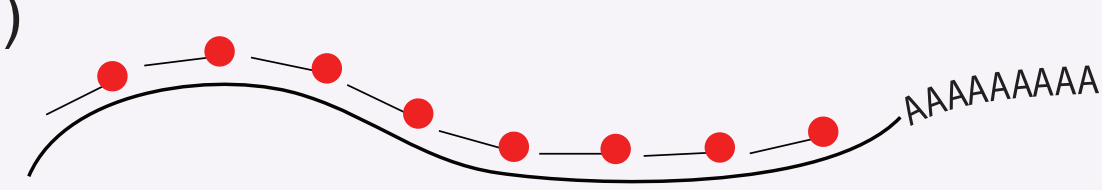

2)
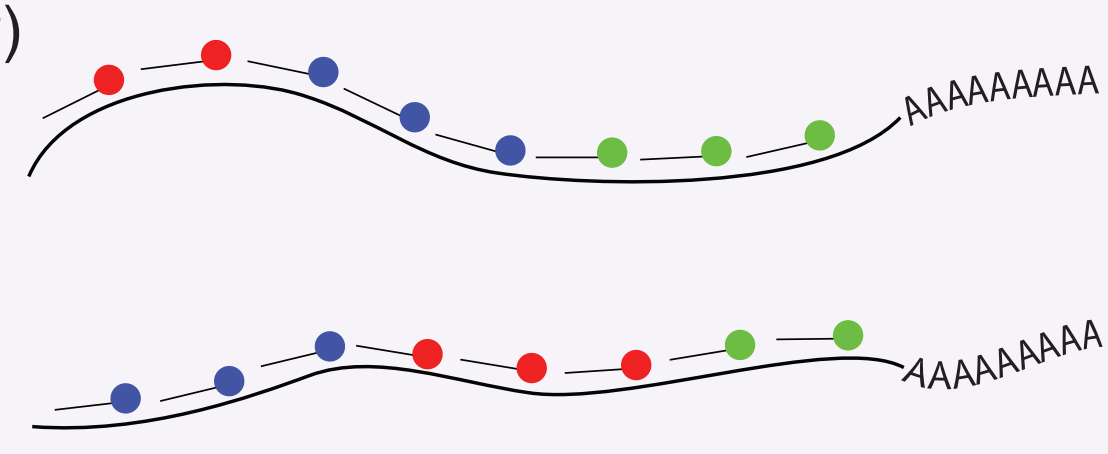

3)
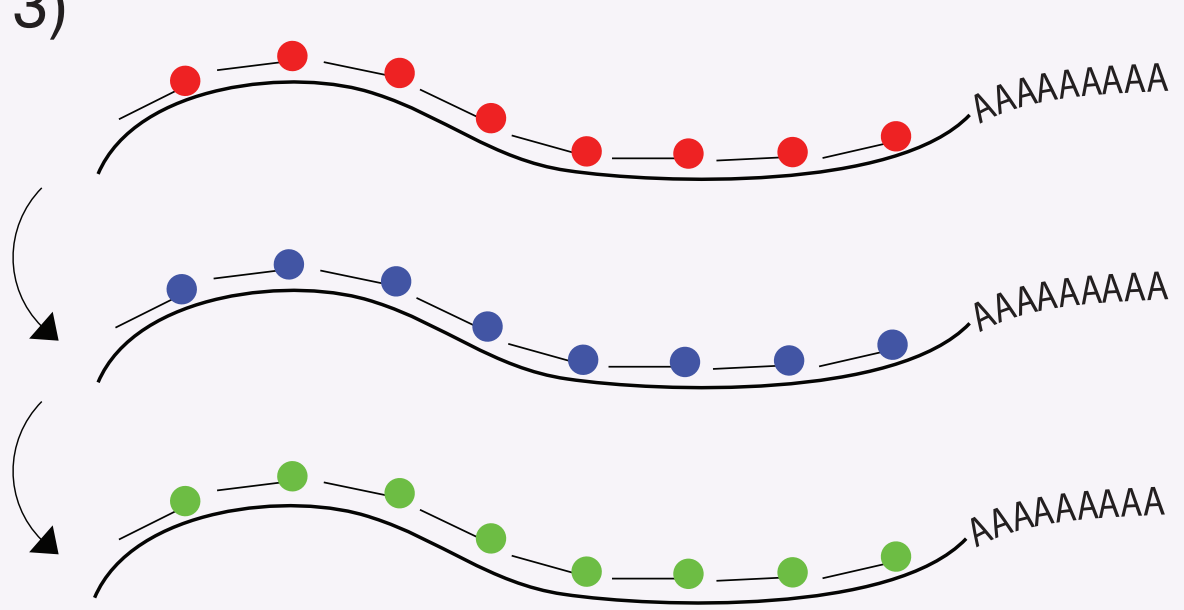

4)

$\smile$ encoding probe

$$
\text { - readout probe (active) }
$$

- readout probe (inactivated)
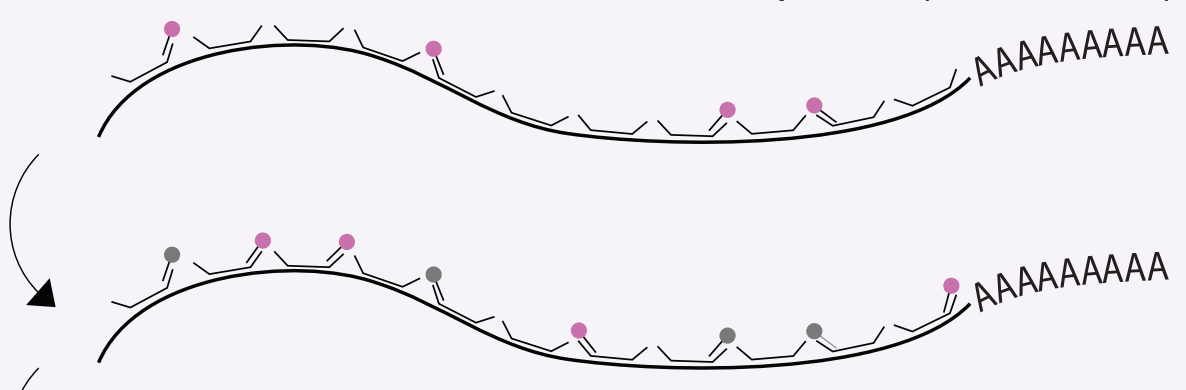

4 i.

\section{B sequencing-based methods}

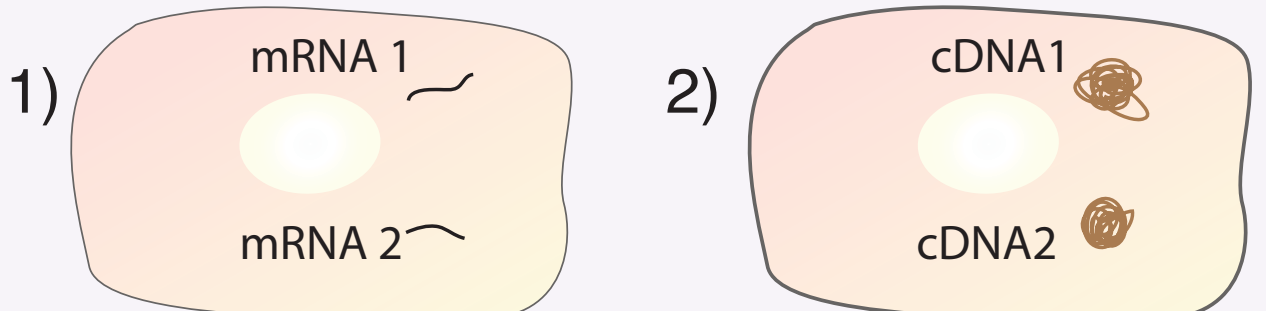

3) cycle 1 cycle 2 cycle 3 cycle 4 cycle $n$

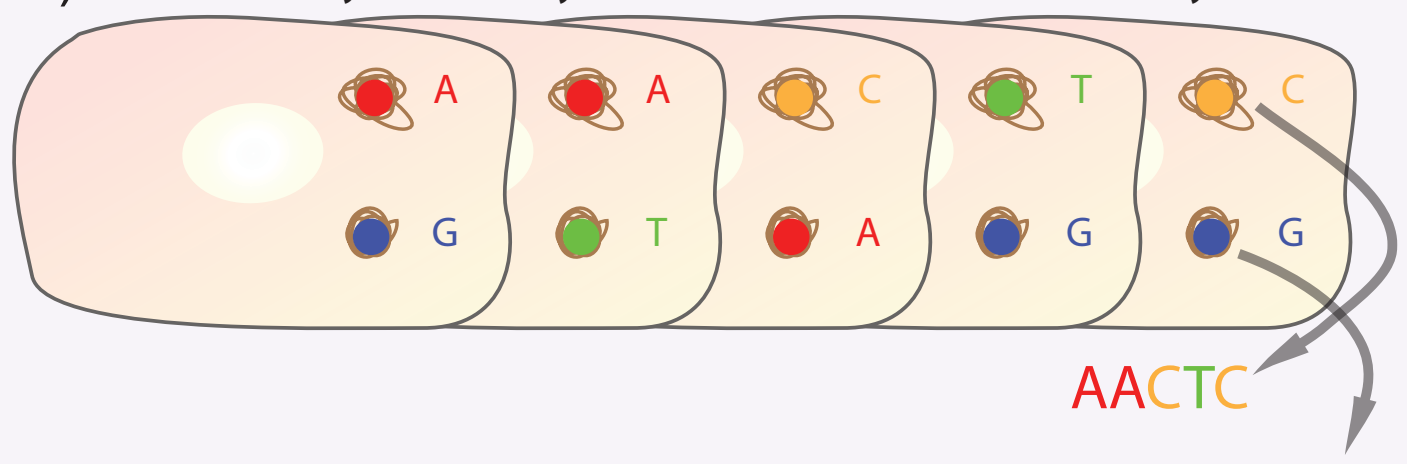

C

1)

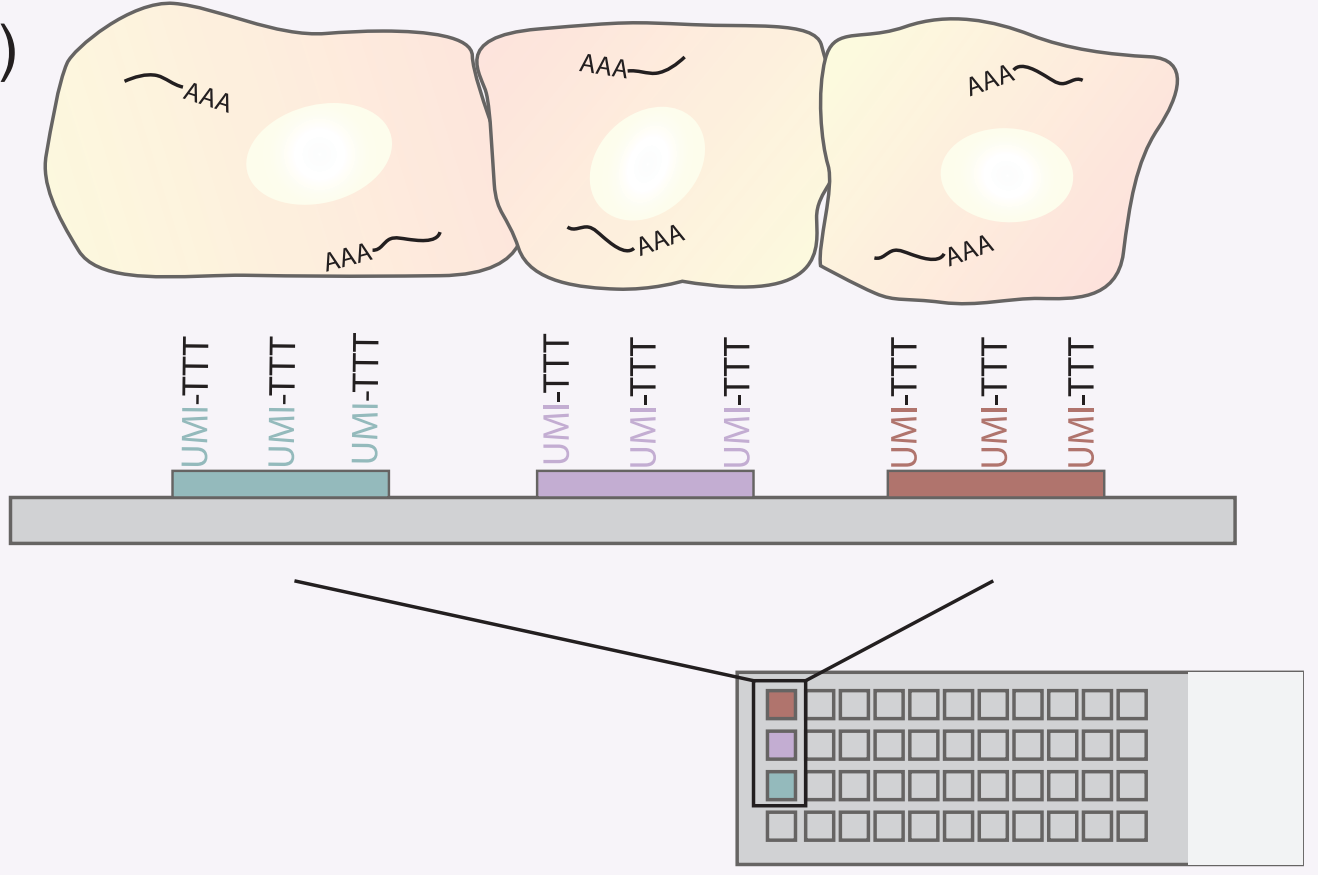

2)

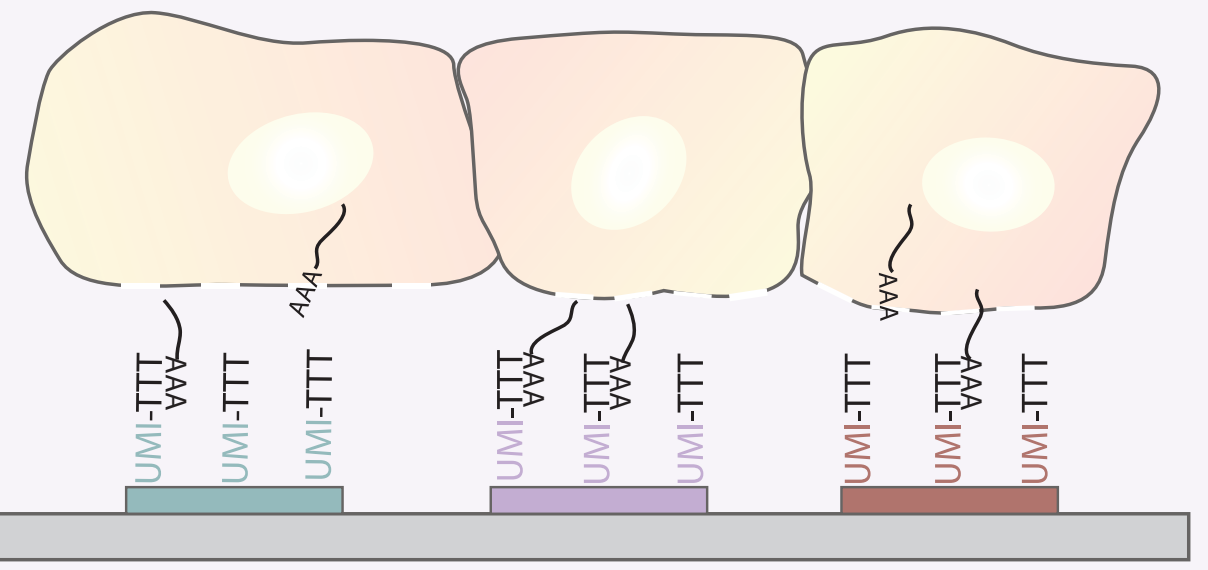

3)

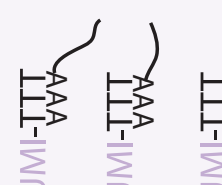

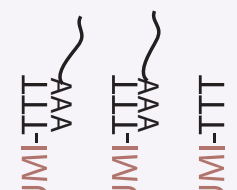
$3<$
D tissue reference map-based methods

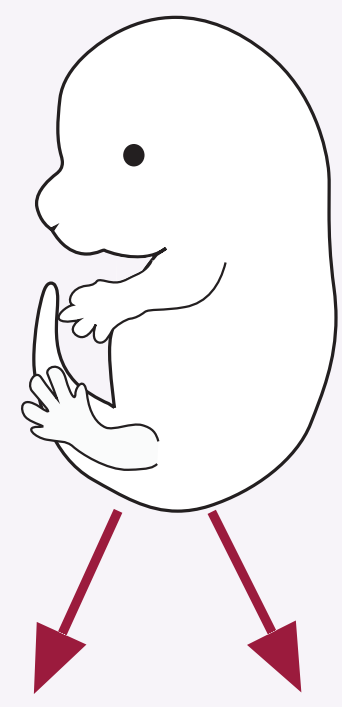

2)
1)

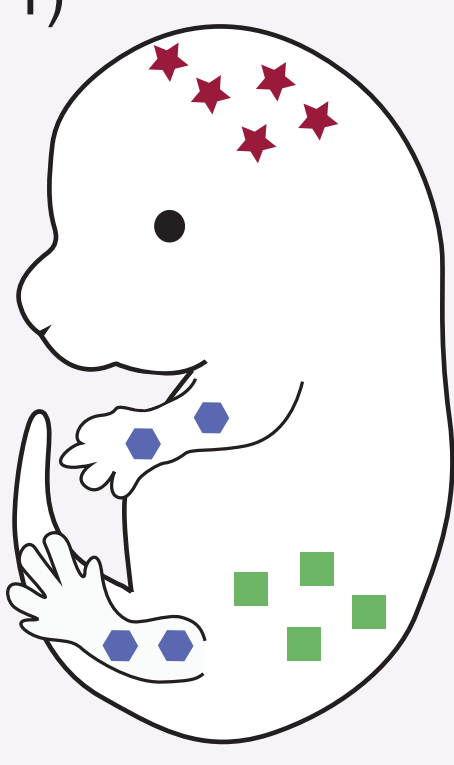

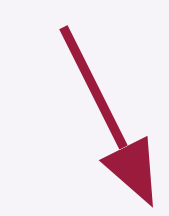

3)

4)

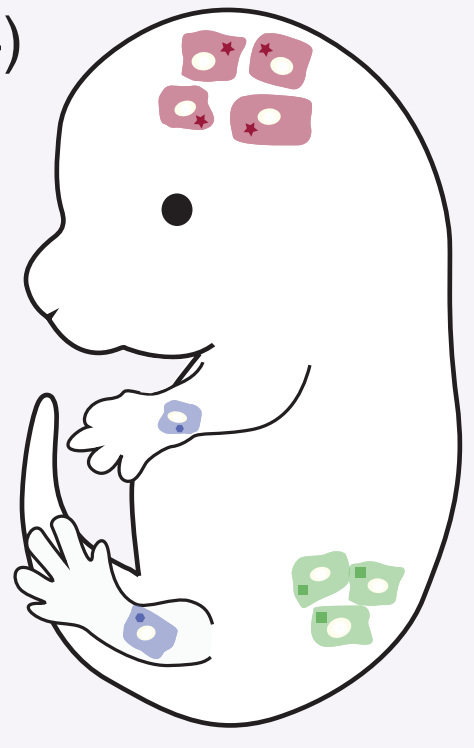

$+$

0

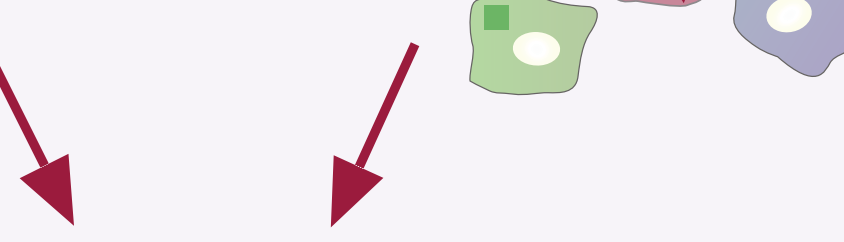

